\title{
A suplementação com óleo de peixe três vezes mais concentrado em epa (ácido eicosapentaenoico) modifica a função renal em ratos
}

\author{
Supplementation with fish oil three times more concentrated in epa (eicosapentaenoic \\ acid) modifies renal function in rats
}

\author{
Bruno Henrique Cassol ${ }^{1}$, Letícia Chaffin Barbosa Peruffo ${ }^{1}$, Sofia Tomaselli Arioni², Ricardo Fernandez ${ }^{3^{*}}$
}

${ }^{1}$ Acadêmico do Curso de Medicina pela Universidade Federal do Paraná (UFPR); ${ }^{2}$ Acadêmico do Curso de Biomedicina pela UFPR; ${ }^{3}$ Médico, Mestre e Doutor em Ciências (Fisiologia Humana), Professor Titular da UFPR

\begin{abstract}
Resumo
Objetivos: o óleo de peixe, rico em ácido eicosapentaenoico (EPA), mostrou benefícios renoprotetores em modelos animais de doenças crônicas. O objetivo deste trabalho foi avaliar os efeitos da suplementação com óleo de peixe três vezes mais concentrado em EPA (EPA 3X) na função renal de ratos e a eficiência desta suplementação por um curto período de tempo. Metodologia: ratos Wistar ( $n=16)$ foram divididos em dois grupos, controle (C) e suplementado (CO) por 14 dias com óleo de peixe (EPA 3X). Foram determinados os seguintes parâmetros: ganho de peso, ingesta de água e ração, fluxo urinário, proteinemia, trigliceridemia, osmolaridade, clearance de creatinina e fração de excreção de sódio. Resultados: os ratos suplementados tiveram níveis de triglicerídeos no plasma $58,4 \%$ menores que os ratos controle $(p=0,0239)$, entretanto os restantes dos parâmetros sistêmicos avaliados não apresentaram alterações. A suplementação com óleo de peixe não alterou o fluxo urinário. Entretanto, no grupo CO identificou-se um aumento na filtração glomerular, com um incremento em média de $67,8 \%$ no clearance de creatinina em relação ao grupo $C(p=0,054)$. A excreção média de proteínas totais na urina e a fração de excreção de sódio foram semelhantes entre os grupos. Conclusão: a suplementação com óleo de peixe três vezes mais concentrado em EPA por um período curto de tempo (quinze dias) provocou alterações significativas tanto em parâmetros sistêmicos (redução significativa na trigliceridemia) quanto na função renal de ratos controle (incremento na filtração glomerular) indicando a eficiência desta suplementação.

Palavras-chaves: Ácido eicosapentaenoico. Filtração glomerular. Função renal. Suplementação com óleo de peixe.
\end{abstract}

\begin{abstract}
Objective: Fish oil, rich in eicosapentaenoic acid (EPA), has shown renoprotective benefits in animal models of chronic diseases. The objective of this work was to evaluate the effects of supplementation with fish oil three times more concentrated in EPA (EPA 3X) on renal function of rats and the efficiency of this supplementation for a short period of time. Methodology: Wistar rats ( $n=16)$ were divided into two groups, control (C) and supplemented (CO), supplemented for 14 days with fish oil (EPA $3 X$ ). The following parameters were determined: weight gain, water and feed intake, urinary flow, proteinemia, triglyceridemia, osmolarity, creatinine clearance and fractional sodium excretion. Results: The supplemented rats had plasma triglyceride levels $58.4 \%$ lower than control rats $(p=0.0239)$, however the rest of the evaluated systemic parameters did not change. Fish oil supplementation did not alter urinary flow. However, in the $\mathrm{CO}$ group an increase in glomerular filtration was identified, with an increase of $67.8 \%$ on average in creatinine clearance compared to group $C(p=0.054$. The mean excretion of total proteins in the urine and the fractional sodium excretion were similar between groups. Conclusion: Supplementation with fish oil three times more concentrated in EPA for a short period of time (fifteen days) caused significant changes both in systemic parameters (significant reduction in triglyceridemia) and in the renal function of control rats (increase in glomerular filtration) indicating the efficiency of supplementation.
\end{abstract}

Keywords: Eicosapentaenoic acid. Glomerular filtration. Renal function. Fish oil supplementation.

\section{INTRODUÇÃO}

O ácido eicosapentaenoico (EPA) e o ácido docosahexaenoico (DHA) são ácidos graxos polinsaturados ômega 3 (AGPI n-3) que têm como precursor o ácido alfa-linolênico (ALA). Já o ácido araquidônico (AA) é um AGPI ômega 6 $(n-6)$ formado a partir do ácido linoleico (AL) (CALDER, 2012; LORENTE-CEBRIÁN et al., 2013). O corpo humano

Correspondente/Corresponding: *Ricardo Fernandez-Centro Politécnico s/n. - Jd. das Américas - Postal Box: 19031 CEP: 81531-990 Curitiba-PR - Telefone: (41) 98434-6536 - E-mail: ricfer@ufpr.br tem uma capacidade limitada de formar EPA e DHA a partir de ALA, portanto os AGPI n-3 devem ser obtidos da ingestão alimentar (CALDER; DECKELBAUN, 2008; LEE; AN, 2013; LORENTE-CEBRIÁN et al., 2013; SIMOPOULOS, 2013; WU et al., 2012). As fontes dietéticas de $A L A$, assim como de AL, são óleos vegetais e as de EPA e DHA são peixes, especialmente tuna, salmão, sardinha e carapau, e também, os vegetais de folhas verde-escuras, e os dois juntos contribuem com 95 a $98 \%$ dos AGPI na dieta de países ocidentais (SIMOPOULOS, 2013; EILANDER et al., 2015). Os AGPI n-3 apresentam efeitos em diversos 
processos fisiológicos na saúde e nas doenças, participam na regulação plasmática de concentrações de lipídeos, na ação insulínica, no desenvolvimento neural e na função visual, e diminui o risco de doenças cardiovasculares, autoimunes e doenças malignas, dentre elas o câncer (BHATTACHARYA et al., 2007; MURRAY et al., 2014; NABAVI et al., 2015). Uma dieta rica em óleo de peixe contendo alto teor de ácidos graxos n-3, como EPA e DHA, apresenta significantes propriedades antiinflamatórias através da inibição de eicosanóides pró-inflamatórios derivados dos ácidos graxos $n-6$, como o AA e a diminuição de citocinas pró-inflamatórias (HONG; LU, 2013; LORENTE-CEBRIÁN et al., 2013; WU et al., 2012). Os AGPI n-3 estão associados à resposta anti-inflamatória, enquanto os AGPI n-6 estão associados à resposta pró-inflamatória (NABAVI et al., 2015). Os eicosanoides pró-inflamatórios derivados do $A A$ - como prostaglandina E2 (PGE2), leucotrienos B4 e tromboxano B2 (TB2) - têm sua produção significativamente reduzida pelos $A G P I n-3$, pois os AGPI n-3 competem com os AGPI n-6 na incorporação de membranas celulares pela ação da ciclooxigenase (COX) e da 5-lipoxigenase (5-LOX) (BHATTACHARYA et al., 2007; CALDER; DECKELBAUN, 2008; CALDER, 2012; LEE; AN, 2013; LOMBARDO; CHICCO, 2006; WU et al., 2012).

Alguns estudos sinalizaram efeito benéfico do óleo de peixe também sobre a função renal em várias patologias tais como dano glomerular, várias formas de insuficiência renal aguda e/ou crônica, nefrotoxicidade por ciclosporina, síndrome hepatorrenal, nefropatia diabética, IgA nefropatia e hipertensão essencial (HARRIS; BREYER, 2016; HAO; BREYER, 2008; CHOU et al., 2012; WU et al., 2012). O óleo de peixe reduziu peroxidação lipídica renal e o dano histológico tubular em ratos diabéticos (JANGALE et al.,2016), assim como diminuiu níveis renais de TB2 e o dano glomerular em um modelo animal de síndrome metabólica (AUKEMA et al., 2013). Dois estudos avaliaram o efeito da suplementação com óleo de peixe sobre a função renal de ratos com CAC e encontraram efeito renoprotetor e aumento do fluxo plasmático renal (FERNANDEZ et al., 2004; COELHO et al., 2012), possivelmente pelo efeito vasodilatador que EPA e DHA produzem ao competirem com o AA pelas enzimas do citocromo $P 450$ (KONKEL; SCHUNCK, 2011). O nosso grupo avalio diversos parâmetros da função renal em ratos portadores do tumor de Walker 256 que tinham sido suplementados por 70 dias na dose de $1 \mathrm{~g} / \mathrm{kg}$ p.c. com óleo de peixe antes ser inoculados com a suspensão de células de tumorais e num modelo de obesidade. Nestes animais observamos melhoras na função e nos marcadores morfológicos renais após a suplementação (COELHO et al., 2012 e 2016).

Embora tenham diversos mecanismos em comum, há indícios de que EPA e DHA tenham efeitos diferentes. Já foi demonstrado que seus efeitos cardiovasculares e anti-aterogênicos diferem (COTTIN et al., 2011). Ensaios clínicos mostraram que o EPA, especificamente, preserva tecido muscular. Além disso, concentrações séricas de EPA foram diretamente relacionadas à massa muscular esque- lética (MURPHY et al., 2010). Desta forma, a utilização de preparações de óleo de peixe com concentrações maiores de EPA poderiam ter efeitos diferentes dos encontrados com as preparações habitualmente empregadas em estudos experimentais.

O objetivo deste trabalho foi avaliar os efeitos da suplementação com óleo de peixe três vezes mais concentrado em EPA sobre a função renal de ratos controle e a eficiência desta suplementação por um curto período de tempo.

\section{METODOLOGIA}

\section{Desenho do estudo}

Foram utilizados ratos machos da linhagem Wistar (Rattus norvegicus, var. albinus), do biotério do Setor de Ciências Biológicas da Universidade Federal do Paraná. Os animais foram submetidos a ciclo invertido claro/escuro (12/12 horas), com temperatura controlada de $22 \pm 1$ 으, com água e ração (Nuvilab AR/ Nuvital Nutrientes Ltda., Curitiba, PR, BRA, composta por $66 \%$ de carboidratos, $23 \%$ de proteínas, $4 \%$ de lipídios, $6 \%$ de fibras e $1 \%$ de vitaminas e minerais) à vontade. Todos os procedimentos envolvendo animais foram aprovados pela Comissão de Ética no Uso de Animais (CEUA) do Setor de Ciências Biológicas da Universidade Federal do Paraná - UFPR (Certificado no 1081).

Dezesseis ratos com 90 dias foram divididos em 2 grupos: C (controle) e CO (suplementado com óleo de peixe três vezes mais concentrado em EPA). O grupo CO foi suplementado via oral todos os dias (técnica de gavagem), por 14 dias, com um composto obtido de uma preparação de lipídeos marinhos contendo 540mg EPA, $110 \mathrm{mg}$ DHA por cada capsula de $1000 \mathrm{mg}$, três vezes mais concentrado em EPA que o óleo de peixe usual, na dose de $1 \mathrm{~g} / \mathrm{Kg}$ de massa corporal/dia (HiOMEGA-3, Naturalis Nutrição \& Farma Ltda., SP, Brasil). Os ratos do grupo C receberam veículo via oral pelo mesmo período de tempo. A variação de pesso corporal dos animais foi monitorada em dias alternados.

No $14^{\circ}$ dia, os animais foram alojados em gaiolas metabólicas individuais (Tecniplast, Italy) para determinação da ingestão de alimento e água e para coleta de urina durante 24 horas. Isso permitiu a monitorização da diurese e determinação de variáveis para avaliação da função renal (concentrações urinárias de creatinina, sódio e proteínas totais). Posteriormente, os animais foram pesados e anestesiados com tiopental intraperitoneal. Foi realizada a coleta de sangue por punção cardíaca para avaliação da osmolaridade e concentrações plasmáticas de creatinina, sódio, potássio, proteínas totais e triacilgliceróis. Após a coleta de sangue, os animais foram eutanasiados por aprofundamento anestésico. 


\section{Análises do plasma e da urina}

Para a dosagem de creatinina plasmática e urinária foi utilizado o método de Jaffe modificado, com kit comercial (Labtest Diagnóstica), em que a creatinina e outros componentes do soro reagem com a solução de picrato em meio alcalino, formando um complexo de cor vermelha, que é medido fotometricamente e que tem resultado expresso em $\mathrm{mg} / \mathrm{dL}$. Esses valores de creatinina urinária e plasmática, juntamente com o fluxo urinário e a massa final do animal, foram utilizados para calcular o Clearance de Creatinina o que fornece um valor equivalente à taxa de filtração glomerular, a qual foi normalizada pela massa corporal.

As determinações da concentração plasmática $\mathrm{Na}^{+} \mathrm{e}$ $\mathrm{K}^{+}$e urinária de $\mathrm{Na}^{+}$foram realizadas por fotometria de chama (Micronal B462, SP, Brasil). Com as concentrações plasmáticas e urinárias de sódio foi determinada a Fração de Excreção (FE) (\%) de $\mathrm{Na}^{+}$.

A determinação da concentração de proteínas totais em sangue foi realizada pela técnica de Biureto, com kit comercial da Katal ${ }^{\circledR}$ para plasma, e a concentração de proteínas totais na urina foi medida pela técnica do complexo Vermelho de Pirogalol-Molibdato, com o kit Proti U/LCR ${ }^{\circledR}$ para as dosagens urinárias, com leitura em espectrofotômetro a $545 \mathrm{~nm}$ e $600 \mathrm{~nm}$, respectivamente. Os dados foram expressos em $\mathrm{g} / \mathrm{dL}$.

A concentração de triglicerídeos no plasma foi mensurada por método enzimático colorimétrico, utilizando o sistema comercial Vida (Vida Biotecnologia, Belo Horizonte-MG), de acordo com as instruções do fabricante. A leitura da absorbância foi realizada em espectrofotômetro a 500nm (Genesys 10UV, Thermo Scientific, USA). Os resultados são expressos em $\mathrm{mg} / \mathrm{dL}$.

A osmolaridade plasmática e urinária foi determinada pelo método de pressão de vapor (MicroOsmômetro Vapro5520 - Vapor Pressure Osmometer, WESCOR-USA). Esses valores foram utilizados para determinar o clearance osmolar e o clearance de água livre (Coelho et al., 2012).

\section{Análise estatística}

Os valores foram expressos como médias \pm erro padrão. Para avaliar o efeito da possível interação e demais efeitos simples da suplementação, foi ajustado um modelo de regressão linear múltiplo. O teste de Kolmogorov-Smirnov foi utilizado para determinar a normalidade da amostra. Foi utilizado o teste $t$ de student não pareado, bicaudal, para comparar os grupos. O critério de significância estatística se obtém com valores de $p<0,05$. O software utilizado para desenvolver a análise foi o GraphPad Prisma 5.0 (GraphPad Inc, San Diego, CA, USA).

\section{RESULTADOS}

\section{Parâmetros sistêmicos}

Os dados sobre o ganho de peso corporal e o consumo de alimento e água se encontram registrados na Tabela
1 e na Figura 1. No primeiro dia de suplementação (ou equivalente para os animais que receberam água), os ratos suplementados tiveram massa corporal semelhante aos ratos controle. Da mesma forma o ganho de peso corporal foi semelhante entre os grupos. A suplementação com óleo de peixe não causou diferenças na ingestão alimentar ou no consumo de água.

Os ratos suplementados com óleo de peixe tiveram níveis de triglicerídeos no plasma $58,4 \%$ menor que os ratos controle $(55,0 \pm 10,6 \mathrm{mg} / \mathrm{dL}$ em $\mathrm{C}$ e $22,9 \pm 7,1 \mathrm{mg} / \mathrm{dL}$ em CO), o que foi significativamente diferente $(p=0,0239)$ (Figura 2). Entretanto, a proteinemia dos ratos suplementados com óleo de peixe tiveram proteinemia média semelhante que os controle: $5,04 \pm 0,22 \mathrm{~g} / \mathrm{dL}$ e $4,80 \pm 0,26 \mathrm{~g} /$ $\mathrm{dL}$, respectivamente.

A natremia e potassemia foram semelhantes entre os grupos. A osmolaridade plasmática tambem não mostrou alterações significativas ( $p>0,05)$ (Tabela 2).

Tabela 1 - Efeito da suplementação com óleo de peixe no peso corporal e no consumo de água e de alimento de ratos controle e suplementados

\begin{tabular}{l|c|c|c}
\hline & C & CO & $p$ \\
\hline Massa corporal (g) no dia 1 & $270,3 \pm 6,5$ & $267,3 \pm 7,8$ & 0,7726 \\
\hline Massa corporal (g) no dia 15 & $327,8 \pm 7,6$ & $324,6 \pm 10,8$ & 0,8173 \\
\hline Crescimento (\%) & $21,3 \pm 1,6$ & $21,4 \pm 1,8$ & 0,9766 \\
\hline Consumo de água (mL/dia) & $33,8 \pm 4,1$ & $29,2 \pm 3,7$ & 0,4251 \\
\hline Consumo de alimento (g/dia) & $21,8 \pm 2,4$ & $20,2 \pm 2,2$ & 0,6477 \\
\hline
\end{tabular}

Fonte: Autoria própria

Figura 1 - Curva de ganho de peso corporal ao longo do tempo (dia 1, $p=0,7726$; dia 15, $p=0,8176$ ).

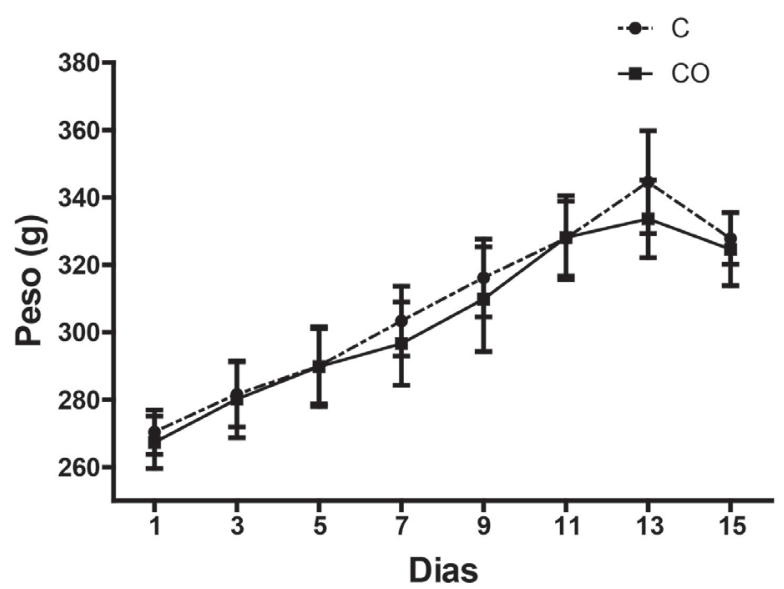

Fonte: Autoria própria 
Figura 2-Concentração plasmática de triglicerídeos. * $p=0,0239$ comparado ao grupo $C$.

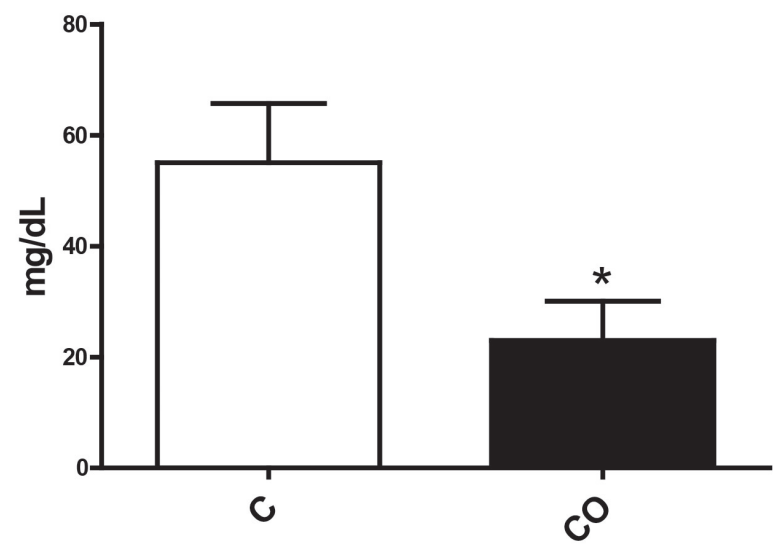

Fonte: Autoria própria

\section{Parâmetros renais}

A suplementação com óleo de peixe não alterou o fluxo urinário. Entretanto, nos animais suplementados identificou-se uma tendência a aumento na filtração glomerular, visto que o grupo CO teve valores em média $67,8 \%$ maiores que o grupo $C$ proximo da significancia estatistica $(p=0,054)$ (Figura 3$)$. A excreção média de proteínas totais na urina foi semelhante entre os grupos. (Tabela 2)

Figura 3 - Filtração glomerular obtida pela determinação do clearance de creatinina e normalizada pelo peso corporal. ${ }^{*} p=0,054$ comparado aos grupos C e CO. (IC 95\%, - 8,783; $0,1071)$

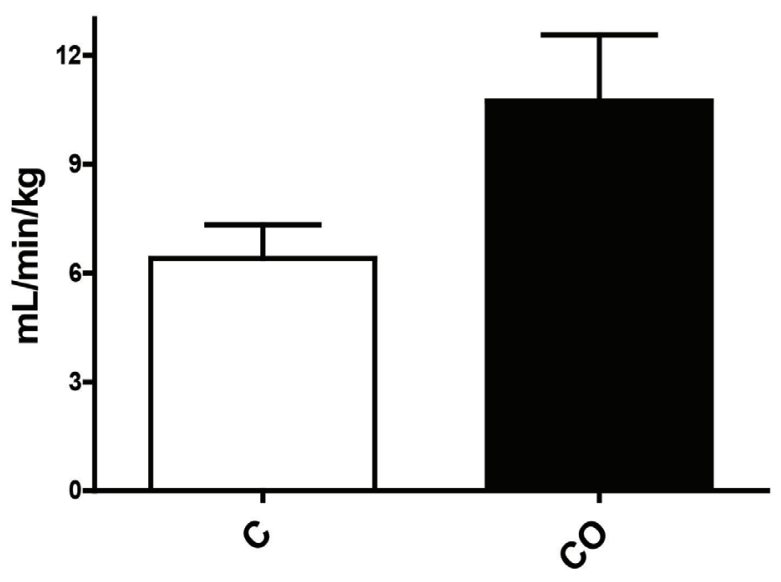

A partir das dosagens plasmáticas e urinárias de $\mathrm{Na}^{+}$e do fluxo urinário foi possível calcular a fração de excreção de $\mathrm{Na}^{+}\left(\mathrm{FE}_{\mathrm{Na}+}\right)$ que estão mostradas na Tabela 2 . A média da $\mathrm{FE}_{\mathrm{Na}+}$ dos animais suplementados foi inferior aos controle, porem sem significancia estatistica. As osmolalidades plasmática e urinária foram dosadas e foram semelhantes entre os grupos. Os valores do clearance de água livre apresentam valores negativos em ambos grupos indicando a reabsorção de água nos segmentos distais do néfron em níveis semelhantes.

Tabela 2 - Efeito da suplementação com óleo de peixe nos parâmetros renais de ratos controle

\begin{tabular}{l|r|r|r}
\hline & \multicolumn{1}{|c|}{$\mathbf{C}$} & \multicolumn{1}{c|}{ CO } & \multicolumn{1}{c}{$\mathbf{p}$} \\
\hline Fluxo urinário (mL/min) & $0,0143 \pm 0,001$ & $0,0123 \pm 0,001$ & 0,2579 \\
\hline Proteinúria (mg/24h) & $15,61 \pm 2,32$ & $13,10 \pm 1,82$ & 0,4041 \\
\hline $\begin{array}{l}\text { Creatinina plasmática } \\
\text { (mg/dL) }\end{array}$ & $0,62 \pm 0,07$ & $0,43 \pm 0,06$ & 0,084 \\
\hline $\begin{array}{l}\text { Clearance de creatinina } \\
\text { normalizado (ml/min/kg } \\
\text { peso corporal) }\end{array}$ & $6,40 \pm 0,92$ & $10,74 \pm 1,81$ & 0,054 \\
\hline Natremia (mEq/L) & $140,8 \pm 0,8$ & $141,3 \pm 0,2$ & 0,6426 \\
\hline Potassemia (mEq/L) & $5,3 \pm 1,0$ & $4,4 \pm 0,7$ & 0,1548 \\
\hline $\begin{array}{l}\text { Fração de excreção de } \\
\text { sódio (\%) }\end{array}$ & $0,80 \pm 0,21$ & $0,53 \pm 0,16$ & 0,3335 \\
\hline $\begin{array}{l}\text { Osmolaridade plasmática } \\
\text { (mOsm/kgH }{ }_{2} \text { ) }\end{array}$ & $283,3 \pm 4,9$ & $288,0 \pm 3,9$ & 0,4664 \\
\hline $\begin{array}{l}\text { Osmolaridade urinária } \\
\text { (mOsm/kg }{ }_{2} \text { O) }\end{array}$ & $1522 \pm 112,6$ & $1620 \pm 111,8$ & 0,5477 \\
\hline $\begin{array}{l}\text { Clearance Osmolar (mL/ } \\
\text { min) }\end{array}$ & $0,066 \pm 0,007$ & $0,060 \pm 0,008$ & 0,6053 \\
\hline $\begin{array}{l}\text { Clearance de água livre } \\
\text { (mL/min) }\end{array}$ & $-0,058 \pm 0,004$ & $-0,054 \pm 0,006$ & 0,6183 \\
\hline $\begin{array}{l}\text { Massa renal normalizada } \\
\text { (g/kg peso corporal) }\end{array}$ & $8,68 \pm 0,35$ & $7,95 \pm 0,48$ & 0,4418 \\
\hline
\end{tabular}

\section{DISCUSSÃO}

A suplementação com óleo de peixe três vezes mais concentrado em EPA por quinze dias provocou alterações em parâmetros sistêmicos e na função renal de ratos controle, indicando a eficiencia desta suplementação mesmo por um curto período de tempo. O óleo de peixe disponível comercialmente provém aproximadamente 300mg dos AGPI n-3 por capsula (CALDER, 2012; LORENTE-CEBRIÁN et al., 2013). Entretanto, no presente trabalho foram utilizadas capsulas contendo 650mg de AGPI n-3, sendo 540mg de EPA o que corresponde a três vezes mais do que é utilizado habitualmente.

Dietas ricas de AGPI n-3 apresentam propriedades anti-inflamatórias e podem apresentar proteção contra aterosclerose e suas consequências, como o infarto do miocárdio e morte súbita. De uma forma geral, seus efeitos benéficos geram redução da concentração dos triacilgliceróis e aumento do $\mathrm{HDL}$, redução do estresse oxidativo, melhora na pressão arterial, na excitabilidade cardíaca, na função plaquetária, endotelial e vascular e no sistema imunitário (COELHO et al., 2012; DIAZ ENCARNACION et al., 2008; LEE; AN, 2013; NOORI et al., 2011). Corroborando outros estudos que utilizaram modelos de suplementação com óleo de peixe, observamos uma redução significativa de $58,4 \%$ na trigliceridemia. Esses dados mostram que a suplementação com óleo de peixe foi assimilada, permitindo afirmar que as demais interações observadas entre o grupo suplementado e o controle se devem aos efeitos do óleo de peixe. 
O sistema enzimático da ciclooxigenase é a via mais importante de metabolização do AA no tecido renal. 0 principal papel fisiológico dos produtos derivados do metabolismo do AA no rim é a modulação da ação de outros autacóides e hormônios, particularmente em relação à ação destas substâncias no tônus vascular, na função glomerular e no manejo tubular de água e eletrólitos (HARRIS; BREYER, 2016; HAO; BREYER, 2008). Os produtos derivados da ação da ciclooxigenase sobre o AA estão relacionados com mudanças observadas na função renal em várias patologias tais como dano glomerular, várias formas de insuficiência renal aguda e/ou crônica, nefrotoxicidade por ciclosporina, síndrome hepatorrenal, nefropatia diabética, nefropatia por IgA e hipertensão essencial (HARRIS; BREYER, 2016; HAO; BREYER, 2008; DIAZ ENCARNACION et al., 2011). Entretanto, a utilização de dietas ricas em AGPIs $n-3$ determina uma competição destes substratos com as vias metabólicas do AA, resultando na formação de produtos finais biologicamente inativos ou pouco potentes. Consequentemente, a suplementação com AGPIs tem sido utilizada para alterar a evolução das patologias renais nas quais os derivados do AA estão envolvidos. Na maioria destas patologias a evolução da doença foi significativamente reduzida pela utilização de um suplemento dietético rico em óleo de peixe que tem papel de suprimir a inflamação seguida de fibrose no tecido renal (BROWN et al., 2000; CHONGVIRIYAPHAN et al., 1999; DIAZ ENCARNACION et al., 2011; DONADIO et al., 2001; HAO; BREYER, 2008; HONG; LU, 2013; LEE; AN, 2013; NOORI et al., 2011; WONG et al., 2010).

$O$ efeito da suplementação com AGPIs n-3 em indivíduos normais tem sido muito menos estudada. No presente estudo a suplementação com óleo de peixe três vezes mais concentrado em EPA gerou um aumento de $67,8 \%$ na VFG $(p=0,054)$. Resultados semelhantes tem sido observados em outros estudos, por exemplo, Schmitz et al. (1991) observou um incremento significativo da filtração por néfron de ratos estudados por micropunção, como consequencia de uma vasodilatação preferencial da arteríola eferente. Um resultado semelhante foi observado num estudo com seres humanos suplementados durante seis semanas com 6g/dia de AGPIs n-3 (DUSSING et al., 1990) . O efeito hemodinâmico observado no grupo suplementado pode ser explicado pela competição ocorrida pelos compostos lipídicos da suplementação na produção de prostanóides moduladores do fluxo plasmático renal, tais como PGE ${ }_{2}$ e ERRO (DOLEGOWSKA et al., 2006). O óleo de peixe, rico em EPA e DHA, gera prostaglandinas e tromboxanos da série 3 e leucotrienos da série 5 , com características vasodilatadoras, diferentemente dos derivados do ácido araquidônico, tais como tromboxanos e prostaglandinas da série 2 e leucotrienos da série 4, que apresentam ações vasoconstritoras (ÁGUILA et al., 2005; HAO; BREYER, 2008). A PGE 2 exerce efeito dilatador na arteríola aferente e, se contrapondo a isto, apresenta papel importante na liberação de renina e indiretamente de angiotensina que têm ações de contração das arteríolas renais, reduzindo o FPR e VFG. Visto este fato, o deslocamento da via de produção de $\mathrm{PGE}_{2}$ - derivado do ácido araquidônico (AGPI n-6) - para produção de $\mathrm{PGE}_{3}$ - derivado do ácido eicosapentaenóico (AGPI n-3) é uma explicação possível para a tendência de incremento na VFG observado no presente estudo.

Valente Gamba et al. (2001) reportaram que a suplementação da dieta em ratos adultos, com $5 \%$ de óleo de peixe, acelerava o progressivo declínio da filtração glomerular e no fluxo plasmático renal habitualmente observado no envelhecimento. Por outro lado, este tratamento incrementou a excreção resultante de ácido nos animais com 9 a 18 meses de idade. Uma deterioração da função renal, proteinúria e queda da filtração glomerular, foram reportadas também por Logan et al. (1992) após uma suplementação por 6 meses com óleo de peixe. No presente estudo não foi observado nenhum efeito deletereo da suplementação, tais como proteínuria, redução da função renal, alteração no manejo renal de sódio ou comprometimento na capacidade de concentração urinária, o que coincide com o observado em estudos anteriores de nosso laboratório (COELHO et al., 2012; COELHO et al., 2016; FERNANDEZ et al., 2004), entretanto os efeitos da suplementação a longo prazo precisam ser estudados.

\section{CONCLUSÃO}

Neste trabalho observamos que a suplementação com óleo de peixe três vezes mais concentrado em EPA por um período curto de tempo (quinze dias) provocou alterações significativas tanto em parâmetros sistêmicos (redução significativa na trigliceridemia) quanto na função renal de ratos controle (aumento na VFG) indicando a eficiência desta suplementação. Desta forma, este modelo é seguro, não apresenta efeitos colaterais e será utilizado para estudar potencias efeitos renoprotetores desta suplementação em patologias crônicas que apresentam repercussão renal, tais como caquexia tumoral e obesidade.

\section{AGRADECIMENTOS}

. À Dra Carolina Arruda Freire, pelo auxilio na determinação da osmolaridade.

\section{REFERÊNCIAS}

AGUILA, M. B. et al. Different edible oil beneficial effects (canola oil, fish oil, palm oil, olive oil, and soybean oil) on spontaneously hypertensive rat glomerular enlargement and glomeruli number. Prostaglandins Other Lipid Mediat., Los Altos, v. 76, p. 74-85, 2005.

AUKEMA, H. M. et al. Dietary fish oil reduces glomerular injury and elevated renal hydroxyeicosatetraenoic acid levels in the JCR:LA-cp rat, a model of the metabolic syndrome. Br. J. Nutr., London, v. 110, n. 1, p. 11-19, 2013.

BHATTACHARYA, A. et al. Different ratios of eicosapentaenoic and docosahexaenoic omega-3 fatty acids in commercial fish oils differentially 
alter pro-inflammatory cytokines in peritoneal macrophages from C57BL/6 female mice. J. Nutr. Biochem., Stonehan, v. 18, p. 23-30, 2007.

BROWN, S. A. et al. Effects of dietary polyinsaturated fatty acid suplementation in early renal insufficiency in dogs. Journal of Laboratory and Clinical Medicine, St. Louis, v. 135, n. 3, p. 275-286, 2000.

CALDER, P. C., DECKELBAUN, R. J. Omega-3 fatty acids: time to get the messages right! Curr. Opin. Clin. Nutr. Metab. Care., London, v. 11, n. 2, p. 91-93, 2008.

CALDER, P. C. Mechanisms of Action of (n-3) Fatty Acids. J. Nutr., London, v. 25, p. 592S-599S, 2012.

CHONGVIRIYAPHAN, N. et al. Effects of tuna fish oil on hyperlipidemia and proteinuria in childhood nephrotic syndrome. J. Med. Assoc. Thai., Bangkok, v. 82, n. 1, p. S122-S128, 1999.

$\mathrm{CHOU}, \mathrm{H}$. H. et al. Omega-3 fatty acids ameliorate proteinuria but not renal function in IgA nephropathy: a meta-analysis of randomized controlled trials. Nephron Clin. Pract., Basel, v. 121, p. c30-c35, 2012.

COELHO, I. et al. Chronic fish oil supplementation partially reverses renal alterations in mice fed with a high-fat diet. J. Funct. Foods, Amsterdam, v. 26, p. 196-207, 2016.

COELHO, I. et al. Fish oil supplementation reduces cachexia and tumor growth while improving renal function in tumor-bearing rats. Lipids, Champaign, v. 47, n. 11, p. 1031-1041, 2012.

COTTIN, S. C. et al. The differential effects of EPA and DHA on cardiovascular risk factors. Proc. Nutr. Soc., London, v. 70, n. 2, p. 215-231, 2011.

DIAZ, E. et al. Signaling pathways modulated by fish oil in salt-sensitive hypertension. Am. J. Physiol. Renal Physiol, Bethesda, v. 294, p. 13231335, 2008.

DOLEGOWSKA, B. et al. Metabolism of eicosanoids and their action on renal function during ischaemia and reperfusion: the effect of alprostadil. Prostaglandins, Leukot. Essent. Fatty Acids, Edinburgh, v. 75, p. 403-411, 2006.

DONADIO, J. V.; GRANDE, J. P. The role of fish oil/omega-3 fatty acids in the treatment of IgA nephropathy. Semin. Nephrol., Philadelphia, v. 24, p. 225-243, 2004.

DUSING, R. et al. Effects of $n-3$ fatty acids on renal function and renal prostaglandin E metabolism. Kidney Int., New York, v. 38, n. 2, p. 315319, 1990.

EILANDER, A. et al. Intake and sources of dietary fatty acids in Europe: Are current population intakes of fats aligned with dietary recommendations? Eur. J. Lipid Sci. Technol., Berlin, v. 117, n. 9, p. 1370-1377, 2015.

FERNANDEZ, R. et al. Effect of chronic fish oil supplementation on renal function of normal and cachectic rats. Braz. J. Med. Biol. Res., Ribeirão Preto, v. 37, p. 1481-1489, 2004.

HAO, C.; BREYER, M. D. Physiological regulation of prostaglandins in the kidney. Ann Rev. Physiol., Palo Alto, v. 70, p. 357-377, 2008.

HARRIS JUNIOR, R. C.; ZHANG, M.; BREYER, R. M. Arachidonic acid metabolites and the Kidney. In: The Kidney. 10. ed. Philadelphia:
Elsevier, 2016.

HONG, S.; LU, Y. Omega-3 fatty acid-derived resolvin and protectins in inflammation resolution and leukocyte functions: targeting novel lipod mediator pathways in mitigation of acute kidney injury. Frontiers in Immunology, New York, v. 4, p. 1-8, 2013.

JANGALE, N. M. et al. Dietary flaxseed oil and fish oil ameliorates renal oxidative stress, protein glycation, and inflammation in streptozotocin-nicotinamide-induced diabetic rats. J. Physiol. Biochem., Pamplona, v. 72, n. 2, p. 327-336, 2016.

KONKEL, A.; SCHUNCK, W. H. Role of cytochrome P450 enzymes in the bioactivation of polyunsaturated fatty acids. Biochim. Biophys. Acta., Amsterdam, v. 1814, n. 1, p. 210-222, 2011.

LEE, S. M.; AN, W. S. Cardioprotective effects of $\omega-3$ of PUFAS in chronic kidney disease. BioMed Res. Int., Hindawi, v. 2013, 1-8, 2013.

LOGAN, J. L. et al.Dietary fish oil interferes with renal arachidonic acid metabolism in rats: correlations with renal physiology. Metabolism, Baltimore, v. 41, n. 4, p. 382-389, 1992.

LOMBARDO, Y. B.; CHICCO, A. G. Effects of dietary polyunsaturated n-3 fatty acids on dyslipidemia and insulin resistance in rodents and humans. A review. J. Nutr. Biochem., Stoneham, v. 17, p 1-13, 2006.

LORENTE-CEBRIÁN, S. et al. Role of omega-3 fatty acids in obesity, metabolic syndrome, and cardiovascular diseases: a review of the evidence. J. Physiol. Biochem., Pamplona v. 69, p. 633-651, 2013.

MURPHY, R.A. et al. Skeletal muscle depletion is associated with reduced plasma (u-3) fatty acids in non-small cell lung cancer patients. J. Nutr., Philadelphia, v. 140, n. 9, p. 1602-1606, 2010.

MURRAY M. $\omega-3$ Polyunsaturated fatty acids and their metabolites as inhibitors of mammalian tumorigenesis. Phytochemistry Reviews, Stuttgart, v. 13, n. 1, p. 139-156, 2014.

NABAVI, S. F. et al. Omega-3 polyunsaturated fatty acids and cancer: lessons learned from clinical trials. Cancer Metastasis Rev., Boston, v. 34, n. 3, p. 359-380, 2015.

NOORI, N. et al. Dietary omega-3 fatty acid, ratio of omega-6 to omega-3 intake, inflammation, and survival in long-term hemodialysis patients. American Journal of Kidney Disease, New York, v. 58, n. 2, p. 248-256, 2011.

SCHMITZ, P. G. et al. Glomerular hemodynamic effects of dietary polyunsaturated fatty acid supplementation. Journal of Laboratory and Clinical Medicine, St. Louis, v. 18, p. 129-135, 1991.

SIMOPOULOS, A. P. Dietary omega-3 fatty acid deficiency and high fructose intake in the development of metabolic syndrome, brain metabolic abnormalities, and non-alcoholic fatty liver disease. Nutrients, Basel, v. 5, p. 2901-2923, 2013.

VALENTE GAMBA, C. et al. Effects of dietary lipids on renal function of aged rats. Brazilian Journal of Medical and Biology Research, Ribeirão Preto, v. 34, p. 265-269, 2001.

WU, J. H. Y. et al. Omega-3 fatty acids and incident type 2 diabetes: a systematic review and meta-analysis. British Journal of Nutrition, London, v. 107, p. 214-227, 2012.

Submetido em: $07 / 08 / 2020$

Aceito em: $14 / 10 / 2020$ 